\title{
Strahlenbelastung durch Bildgebung nicht ohne
}

\begin{abstract}
Die Strahlenbelastung der Bevölkerung durch medizinische Bildgebung ist im Ansteigen und erreicht bei einem beträchtlichen Anteil mindestens mäßig effektive Dosen zwischen 3 und $20 \mathrm{mSv} / \mathrm{Jahr}$.
\end{abstract}

- In einer retrospektiven Kohortenstudie wurden in den Regionen Arizona, Dallas, Orlando, Florida und Wisconsin zwischen 1. Januar 2005 und 31. Dezember 2007 die kumulativen effektiven Strahlendosen von 952420 Personen zwischen 18 und 64 Jahren errechnet. In diesem Zeitraum unterzogen sich 655613 Versicherte $(68,8 \%)$ mindestens einem bildgebenden medizinischen Verfahren mit Strahlenexposition. Die durchschnittliche kumulative effektive Dosis durch die Bildgebung betrug 2,4 $\pm 6,0 \mathrm{mSv}$ pro Versicherten und Jahr.

Einer moderaten effektiven Dosis zwischen 3 und $20 \mathrm{mSv}$ waren 193,8 pro 1000 Versicherten und Jahr ausgesetzt. Dies entspricht der Größenordnung, wie sie bei berufsmäßig strahlenexponierten Personen im Gesundheitswesen und in der Nuklearindustrie, die sich unter laufender Kontrolle befinden, erreicht werden darf. Bei Patienten erfolgt diese Kontrolle allerdings nicht.

Hohe effektive Expositionen zwischen 20 und $50 \mathrm{mSv}$ wurden bei 18,6 und sehr hohe Belastungen über 50 $\mathrm{mSv}$ bei 1,9 pro 1000 Versicherten und Jahr errechnet. Im Allgemeinen beobachtete man eine Zunahme der Strahlendosis mit zunehmendem Lebensalter, wobei Frauen noch stärker belastet waren als Männer. Computertomografien und nuklearmedizinische Untersuchungen trugen zu 75,4\% zur kumulativen effektiven Dosis bei. $81,8 \%$ der gesamten Strahlendosis wurde bei ambulanten Untersuchungen appliziert.
KOMMENTAR: Nachdem die Studie nur auf Abrechnungsdaten einer Krankenversicherung fußt, könnte es durchaus sein, dass die tatsächliche Strahlenexposition der Bevölkerung durch medizinische Maßnahmen noch viel höher ist. Es wurde nämlich nur die Dosis von Standarduntersuchungen errechnet, die bei optimalen technischen Voraussetzungen appliziert wird. Nachdem bekannt ist, dass selbst innerhalb derselben Institution die Strahlenbelastung beim CT in Abhängigkeit von zahlreichen technischen Details variieren kann, könnte die Wirklichkeit noch ungünstiger aussehen.

H. S. FüEßL =

\section{- R. Fazel et al. \\ Exposure to low-dose ionizing radiation from medical imaging procedures. New Engl. J. Med. 361 (2009) 9, 849-857}

\section{Hier sehen Sie der Krise ins Auge}

— Eine 37-jährige Frau litt seit drei Tagen unter Kopfschmerzen und Sehstörungen. Ihr Blutdruck betrug 220/150 mmHg. Bei der Visusprüfung konnte sie nur Finger zählen, und die Pupillenreaktion war träge.

Die Funduskopie ergab ein beidseitiges Papillenödem, Cot-
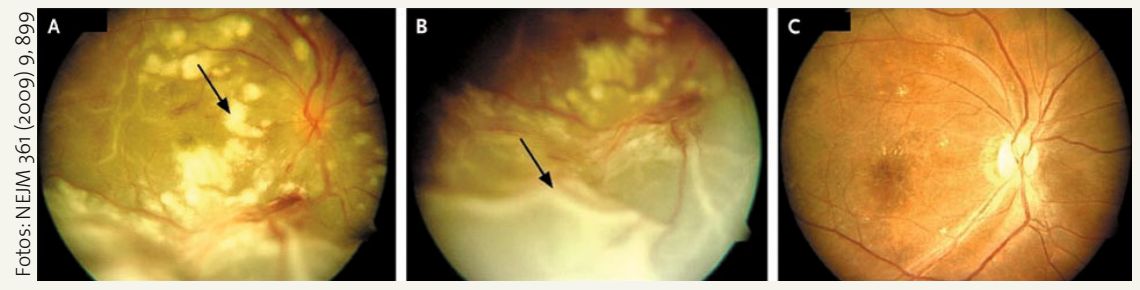

A: Papillenödem, Cotton-wool-Exsudate; B: beginnende Netzhautablösung; C: Zustand nach Behandlung ton-wool-Exsudate (Abb. A, Pfeil) und beidseits eine untere seröse Netzhautablösung (Abb. B). Bei weiteren Untersuchungen ergab sich, dass die Patientin eine hochgradige Niereninsuffizienz mit einem Kreatinin von $14,9 \mathrm{mg} / \mathrm{dl}$ hatte.

Sie wurde mit Isosorbid-Dinitrat und Amlodipin behandelt und hämodialysiert. Nach einer Woche betrug der Blutdruck 140/90 mmHg. Der Visus hatte sich beidseits auf 20/80 gebessert. Die Funduskopie ließ einen weitgehenden Rückgang der Netzhautablösung und der Zahl der Cotton-wool-Exsudate erkennen. Zwei Wochen nach Beginn der Therapie hatte sich der Visus weiter bis auf 20/30 gebessert. Die Retinaveränderungen waren vollständig zurückgebildet (Abb. C). Die Nierenerkrankung befindet sich derzeit noch in der Abklärung. Ein Netzhautödem und eine exsudative Netzhautablösung gehören zu den Definitionskriterien einer hypertensiven Krise, die notfallmäßig behandelt werden muss.

-I. Tajunisah, D. K. Patel

H. S. FÜEßL

(University of Malaysia, Kuala Lumpur 50603, Malaysia. Retinal detachment in malignant hypertension. New Engl. J. Med. 361 (2009) 9,899 